\title{
Video Article \\ EPA Method 1615. Measurement of Enterovirus and Norovirus Occurrence in Water by Culture and RT-qPCR. II. Total Culturable Virus Assay
}

\author{
G. Shay Fout ${ }^{1}$, Jennifer L. Cashdollar ${ }^{1}$ \\ ${ }^{1}$ National Exposure Research Laboratory, U.S. Environmental Protection Agency
}

Correspondence to: G. Shay Fout at fout.shay@epa.gov

URL: https://www.jove.com/video/52437

DOI: doi:10.3791/52437

Keywords: Environmental Sciences, Issue 115, virus, infectious, waterborne, concentration, detection, occurrence, total culturable virus assay Date Published: 9/11/2016

Citation: Fout, G.S., Cashdollar, J.L. EPA Method 1615. Measurement of Enterovirus and Norovirus Occurrence in Water by Culture and RT-qPCR. II. Total Culturable Virus Assay. J. Vis. Exp. (115), e52437, doi:10.3791/52437 (2016).

\section{Abstract}

A standardized method is required when national studies on virus occurrence in environmental and drinking waters utilize multiple analytical laboratories. The U.S Environmental Protection Agency's (USEPA) Method 1615 was developed with the goal of providing such a standard for measuring Enterovirus and Norovirus in these waters. Virus is concentrated from water using an electropositive filter, eluted from the filter surface with beef extract, and then concentrated further using organic flocculation. Herein we present the protocol from Method 1615 for filter elution, secondary concentration, and measurement of total culturable viruses. A portion of the concentrated eluate from each sample is inoculated onto ten replicate flasks of Buffalo Green Monkey kidney cells. The number of flasks demonstrating cytopathic effects is used to quantify the most probable number (MPN) of infectious units per liter. The method uses a number of quality controls to increase data quality and to reduce interlaboratory and intralaboratory variation. Laboratories must meet defined performance standards. Method 1615 was evaluated by examining virus recovery from reagent-grade and ground waters seeded with Sabin poliovirus type 3. Mean poliovirus recoveries with the total culturable assay were $111 \%$ in reagent grade water and $58 \%$ in groundwaters.

\section{Video Link}

The video component of this article can be found at https://www.jove.com/video/52437/

\section{Introduction}

Enteric viruses are a diverse group of viruses that infect the human intestinal system and which are transmitted via the fecal-oral route. These viruses enter surface and ground waters through sewage treatment plant and septic tank effluents, improperly designed or broken septic tanks, broken sewer lines, combined sewer overflows, and other point and non-point sources ${ }^{1-4}$. Human infections and disease from waterborne viruses occur via consumption of contaminated or inadequately disinfected water or through recreational water contact. Disease symptoms may involve mild to severe gastroenteritis; conjunctivitis; fever; upper respiratory distress; hand, foot and mouth disease; myocarditis; aseptic meningitis; encephalitis; paralysis; sepsis ${ }^{5-8}$, and death ${ }^{9,10}$.

USEPA Method 1615 provides a procedure to measure infectious enteric virus particles in environmental and drinking waters. These waters can contain a mixture of infectious and non-infectious virions, but only the infectious particles pose a potential health hazard. Infectious virus particles lose infectivity over time in environmental and drinking waters from loss of protein capsid integrity, damage to nucleic acids due to UV radiation from sunlight, and damage due to any disinfectants that may be present ${ }^{11-13}$. The total culturable virus procedure provided in the method is based upon the production of cytopathic effects (CPE) in the Buffalo Green Monkey kidney (BGM) cell line. This cell line was chosen because of its widespread use in the environmental virology field ${ }^{14,15}$, even though the range of infectious virus types detected are restricted primarily to certain enteroviruses $^{15}$. The purpose of this paper is to describe Method 1615's procedures for elution of five-inch electropositive cartridge filters, secondary concentration, and measurement of total culturable viruses. An evaluation of the overall method is described in Cashdollar et al. ${ }^{16}$.

\section{Protocol}

NOTE: Please see supplemental materials section S1 for a list of definitions. The QA procedures associated with USEPA Method 1615 are described in the supplemental materials section S2.

\section{Filter Elution Procedure}

\section{First Elution}

1. Place $500 \mathrm{ml}$ of buffered $1.5 \%$ beef extract, $\mathrm{pH} 9.0$, warmed to room temperature in a graduated cylinder. Open the filter cartridge housing and add a sufficient amount of beef extract to cover the electropositive filter completely. Replace the filter housing lid and pour the remaining beef extract into a sterile beaker. 
2. After $1 \mathrm{~min}$ of contact time, pass the beef extract solution in the housing along with that remaining in the sterile beaker slowly through the filter using a pressure container or peristaltic pump. Collect the eluate into a $2 \mathrm{~L}$ glass beaker.

2. Second elution

1. Repeat Steps 1.1 using an additional $500 \mathrm{ml}$ of buffered $1.5 \%$ beef extract, but increase the contact time at Step 1.1 .2 to $15 \mathrm{~min}$.

2. Add the beef extract from the second elution to the $2 \mathrm{~L}$ beaker containing that from the first elution. Add a sterile stir bar to the beaker.

\section{Organic Flocculation Concentration Procedure}

1. Sterilize a combination-type $\mathrm{pH}$ electrode with $0.525 \%$ sodium hypochlorite for at least $5 \mathrm{~min}$. Rinse the electrode with sterile $\mathrm{dH}_{2} \mathrm{O}$ and then dechlorinate with $0.05 \mathrm{M}$ sodium thiosulfate. Calibrate the $\mathrm{pH}$ meter using $\mathrm{pH} 4$ and 7 standards.

2. Place the beaker containing the eluate on a stir plate. Turn on the plate and increase the stirring speed until a vortex is formed.

3. Adjust the $\mathrm{pH}$ of the eluate to $3.5 \pm 0.1$ slowly by drop wise addition of $1.2 \mathrm{M} \mathrm{HCl}$ to the eluate. Add the $\mathrm{HCl}$ drop-wise because rapid addition will inactivate virus. During this time the eluate will become cloudy as a precipitate begins to form.

4. Reduce the mixing speed to a slow stir and then continue to monitor and maintain the $\mathrm{pH}$ of the eluate at $3.5 \pm 0.1$ at room temperature for 30 $\min$.

5. Pour the precipitated beef extract suspension into one or more centrifuge bottles and centrifuge for $15 \mathrm{~min}$ at $2,500 \times \mathrm{g}$ at $4{ }^{\circ} \mathrm{C}$. Remove the bottles from the centrifuge and either aspirate or slowly decant the supernatant to prevent the loss of the pelleted precipitate. Discard the supernatant.

NOTE: There can be considerable variation among beef extract lots in the quantity and quality of the precipitate. The precipitate produced from some lots will dissolve quickly while that from other lots dissolves with difficulty.

6. Add $30 \mathrm{ml}$ of $0.15 \mathrm{M}$ sodium phosphate to the centrifuge bottle containing the precipitate.

1. Use $0.15 \mathrm{M}$ sodium phosphate, $\mathrm{pH} 9.0$ for precipitates from beef extract lots that dissolve within $5 \mathrm{~min}$. Stir for 10 min after the precipitate is completely dissolved, and then go immediately to Step 2.7 .

2. Use $0.15 \mathrm{M}$ sodium phosphate, $\mathrm{pH} 7.0-7.5$ for all other precipitates. Stir for 10-15 min to dissolve, or for more difficult precipitates, break them up with a sterile spatula, by repeatedly drawing the solution up and down during the stirring with a pipette, by shaking the precipitate at $160 \mathrm{rpm}$ on an orbital shaker, or by a combination of these procedures.

NOTE: When using more than one centrifuge bottle, combine the precipitates using less than $30 \mathrm{ml}$ of sodium phosphate and then use the remaining portion of the $30 \mathrm{ml}$ to rinse the bottles after combining the precipitates into one bottle or beaker.

3. If the combined precipitate is in a flat bottom centrifuge bottle, add a stir bar to the bottle. Go to Step 2.6.5.

4. If the combined precipitate is in a centrifuge bottle with a conical bottom, transfer it into a small glass beaker and add a stir bar to the beaker.

5. Place the bottle or beaker onto a magnetic stirrer, and stir until the precipitate has dissolved completely.

6. Re-sterilize a combination type $\mathrm{pH}$ electrode with $0.525 \%$ sodium hypochlorite and dechlorinate with $0.05 \mathrm{M}$ sodium thiosulfate as described in Step 2.1. Calibrate the $\mathrm{pH}$ meter using $\mathrm{pH} 7$ and 10 standards. Slowly adjust the $\mathrm{pH}$ of the completely dissolved precipitate to 9.0 with $1 \mathrm{M} \mathrm{NaOH}$ and then stir for $10 \mathrm{~min}$.

7. Remove the stir bar and centrifuge the dissolved precipitate for $10 \mathrm{~min}$ at $4,000-10,000 \times \mathrm{g}$ and $4{ }^{\circ} \mathrm{C}$.

8. Carefully pour the supernatant into a glass beaker without disturbing the pellet. Add a stir bar to the beaker and discard the pellet.

9. Place the beaker onto a magnetic stirrer, and stir the solution. Add $1.2 \mathrm{M} \mathrm{HCl}$ drop wise to adjust the $\mathrm{pH}$ to $7.0-7.5$.

10. Filter sterilize the supernatant by passage through a sterilizing filter containing a prefilter that has been pretreated with $15 \mathrm{ml}$ of $1.5 \%$ beef extract, $0.05 \mathrm{M}$ glycine, $\mathrm{pH}$ 7.0-7.5.

11. Assay Sample Volume (S) calculations:

1. Use Equation 1 to calculate $S$ for all test samples except the Lab Fortified Blank and the Lab Reagent Blank,

$S=\frac{D}{T S V} \times F C S V \quad$ Equation 1

where D (Volume of Original Water Sample Assayed) is $500 \mathrm{~L}$ for groundwater, TSV (Total Sample Volume) is the volume of field sample passed through the 5 -inch electropositive cartridge filter received by the laboratory, and FCSV (Final Concentrated Sample Volume) is the volume following filtration in Step 2.10. An example is shown in supplemental materials Section S4.1.

2. Calculate $S$ for the Lab Fortified Blank (LFB; i.e., a positive quality control using seeded reagent grade water) and the Lab Reagent Blank (LRB; i.e., a negative quality control using reagent grade water) by multiplying the FCSV by 0.3 .

12. Divide the FCSV into three subsamples.

1. Prepare subsamples 1 and 2 with a volume equal to 1.04 times the Assay Sample Volume. Freeze these subsamples at or below -70 ${ }^{\circ} \mathrm{C}$ if they cannot be analyzed using the total culturable virus assay (Subsample 1, Step 4) or processed for the molecular assays (not shown) within $24 \mathrm{hr}$; otherwise, hold at $4{ }^{\circ} \mathrm{C}$.

2. Freeze the remaining volume (subsample 3 ) at or below $-70^{\circ} \mathrm{C}$.

13. Calculate the Inoculum Volume by dividing the $S$ by 10 .

\section{Total Culturable Virus Quantal Assay}

NOTE: For all steps always add solutions carefully to avoid disturbing the cell monolayer.

1. Decant or aspirate media from test vessels containing a monolayer of BGM cells at 3-6 days post splitting and then add a volume of balanced salt solution equal to the media removed.

2. Decant or aspirate the balanced salt solution from the cell culture test vessels being used and then inoculate the cell culture test vessels 
1. Inoculate 10 test vessels for each test sample with a volume of subsample 1 equal to the Inoculum Volume along with the total culturable virus quantal assay controls (supplemental materials section S2.4).

2. For the LFB and the Lab Fortified Sample Matrix (LFSM; i.e., seeded water matrix sample), prepare 5-, 25-, and 125-fold dilutions using subsample 3 and $0.15 \mathrm{M}$ sodium phosphate, $\mathrm{pH} 7.0-7.5$ as a diluent. An example of a procedure for making the dilutions is given in supplemental materials section S3. Inoculate 10 washed cell culture test vessels for each dilution series using an Inoculum Volume on each test vessel in addition to the vessels inoculated with undiluted Subsample 1 in Step 3.2.1.

3. For any test sample from Step 3.2.1 (other than those inoculated in Step 3.2.2) that has CPE in all 10 replicates after 14 days of incubation (see Step 3.3), prepare 5-, 25-, and 125-, and 625-fold dilutions of subsample 3. Inoculate 10 washed cell culture test vessels for each dilution series using an Inoculum Volume on each test vessel along with a new set of the total culturable virus quantal assay controls (supplemental materials section S2.4).

4. Distribute the inoculum over the surface of the cell monolayers by tilting the vessels back and forth. Incubate the test vessels at room temperature for $80-120 \mathrm{~min}$ on a mechanical rocking platform at 1-5 oscillations/min or with rocking of the vessels every $15-20 \mathrm{~min}$ to allow any virus present to adsorb to cells.

5. Add prewarmed maintenance medium, and then incubate the test vessels at $36.5 \pm 1{ }^{\circ} \mathrm{C}$.

3. Look for the appearance of CPE in each test vessel using a microscope daily for the first $3 \mathrm{~d}$ and then examine them every $2-3$ days up to day 14. Transfer any test vessels that show $\geq 75 \% \mathrm{CPE}$ to a freezer set at or below $-70{ }^{\circ} \mathrm{C}$. Freeze all remaining cultures and the total culturable virus quantal assay controls at or below $-70{ }^{\circ} \mathrm{C}$ after examining the vessels on the last day.

4. Thaw all the cultures and filter $\geq 15 \%$ of the medium from every CPE-positive test vessel through a $0.2 \mu \mathrm{m}$ sterilizing filter. If the specified volume cannot be passed through the filter due to clogging, centrifuge the medium for $10 \mathrm{~min}$ at $1,500-18,000 \times \mathrm{g}$ and $4{ }^{\circ} \mathrm{C}$ prior to filtration.

5. Perform a second passage of all $1^{\text {st }}$ passage test vessels using washed BGM test vessels.

1. Inoculate the new test vessels with an inoculation volume that represents $10 \%$ of the thawed medium from all negative test vessels and from the filtered medium from positive vessels.

2. Repeat Steps 3.2.4-3.4.3, but freeze any test vessel that was negative on the $1^{\text {st }}$ passage and positive on the $2^{\text {nd }}$ as described in Step 3.3. Perform a $3^{\text {rd }}$ passage as described for the $2^{\text {nd }}$ passage using only the negative assay controls and cell cultures that were negative during the $1^{\text {st }}$ passage and positive in the $2^{\text {nd }}$ passage.

6. Identify individual test vessels as virus positive when they show CPE in both the $1^{\text {st }}$ and $2^{\text {nd }}$ passages or, in the case where CPE does not occur until the $2^{\text {nd }}$ passage, in both the $2^{\text {nd }}$ and $3^{\text {rd }}$ passages.

7. Use USEPA's Most Probable Number Calculator with the default program settings set as shown in Table $\mathbf{S} 2$ to calculate the virus titers of al test samples.

1. Input the number of virus positive replicate test vessels from Step 3.6 for each test sample into the calculator to determine the MPN/ml value $\left(M_{m L}\right)$ and the upper $\left(C L_{u m L}\right)$ and lower $\left(C L_{/ m L}\right) 95 \%$ confidence limits $/ \mathrm{ml}$ values.

2. Obtain the MPN/L value $\left(M_{L}\right)$ of the corresponding test sample using Equation 2.

$M_{L}=\frac{M_{m_{L}} S}{D} \quad$ Equation 2

$M_{m L}$ is the MPN/ml value in Step 3.7, $S$ is the Assay Sample Volume, and $D$ is the Volume of Original Water Sample Assayed.

3. Calculate the upper confidence limit/L by substituting the $C L_{u m L}$ value for the $M_{m L}$ value. Calculate the lower confidence limit/L by substituting the $\mathrm{CL}_{\mathrm{ImL}}$ value for the $\mathrm{M}_{\mathrm{mL}}$ value. An example calculation is shown in supplemental materials Section S4.2.

4. Report $M_{m L}$ values of 0 as $\leq 1 / \mathrm{D}$. For example, $\leq 0.002 \mathrm{MPN} / \mathrm{L}(\leq 1 / 500 \mathrm{~L})$ for groundwater samples.

5. Calculate the MPN and 95\% confidence limit values for each Lab Fortified Blank and Lab Reagent Blank by first multiplying the values/ $\mathrm{ml}$ obtained in the calculator by $S$ and then dividing the result by 0.3 .

\section{Representative Results}

Virus was concentrated from the source groundwater of three drinking water treatment plants and a private well using electropositive filters. Two sample sets, consisting of a field sample and LFSM control, were collected from the treatment plants on separate occasions, and one sample set was collected from the private well. Overall virus recovery was determined using LFSM samples from two of the plants and the private well (two samples from one plant and one sample from another were excluded from the calculation because the MPN value of the seed used with each sample could not be determined accurately due to abnormal CPE results among replicate flasks). Mean poliovirus recovery from groundwater samples averaged $58 \%$ with a coefficient of variation of $79 \%$ (Figure 2$)^{16}$. No culturable virus was detected in any of the duplicate unseeded ground water field samples.

Method performance also was measured using LFB samples modified by using two different seed levels. A "low" titer of 300 MPN of poliovirus was used to evaluate performance at levels less than the "standard" 500 MPN LFB level used in USEPA Method 1615. A "high" titer of 1,000 MPN of poliovirus was used to test performance at the level of the LFSM control. These controls performed similarly with a mean recovery of $111 \%$ and a coefficient of variation of $100 \%$ (Figure 2). All LRB samples were negative and all LFB samples performed within the acceptance range (supplemental materials Table S1). 


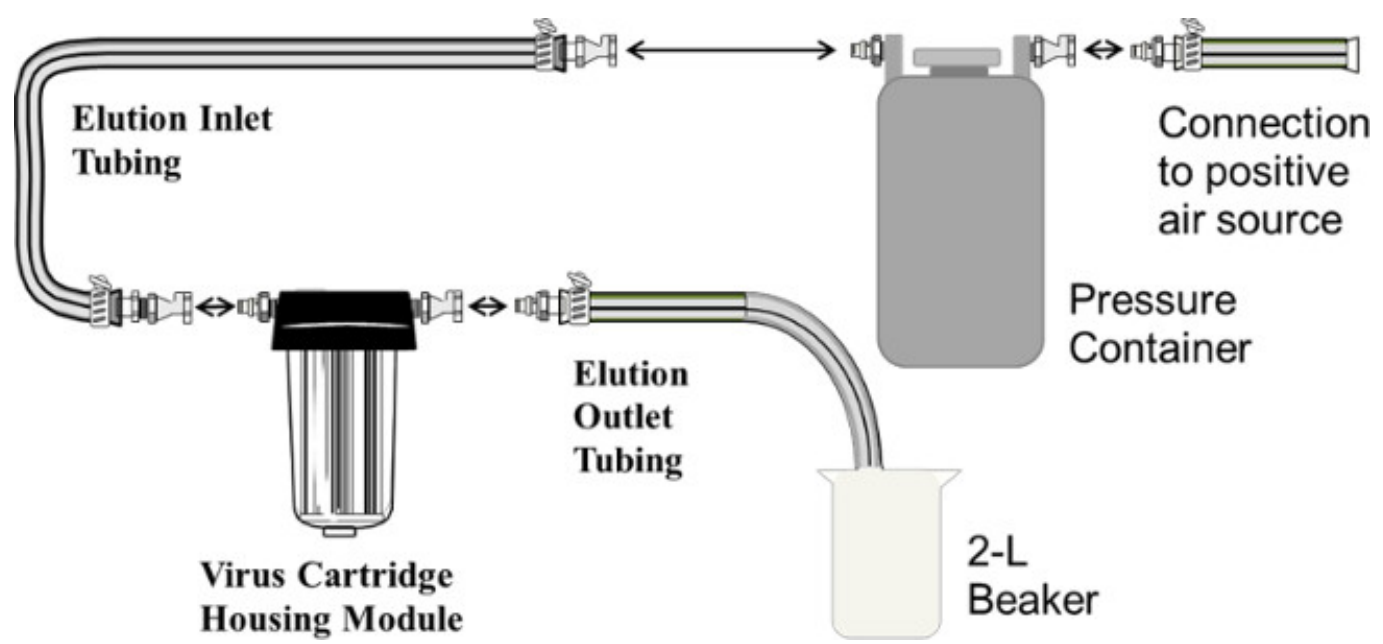

Figure 1. Filter Elution. A schematic for using a pressure container for cartridge filter elution is shown. Positive air pressure is used to push beef extract solution in the pressure container through the cartridge housing containing the electropositive cartridge filter. A peristaltic pump can be substituted for the pressure container, with the inlet of the pump being placed in the container holding the beef extract solution.

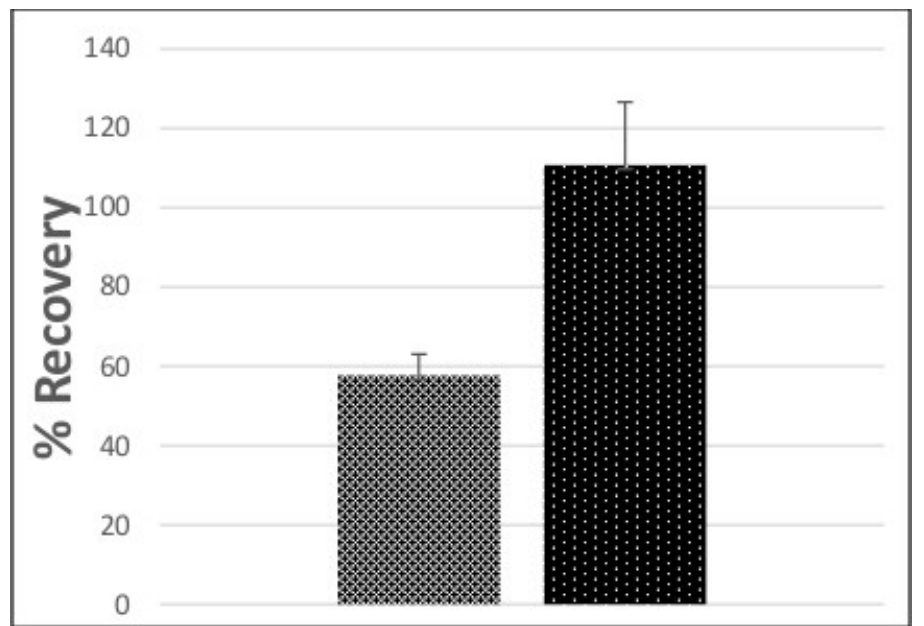

Figure 2. Mean Poliovirus Recovery (\%) from Ground and Reagent-Grade Water. The mean percent recovery is shown for poliovirus from ground ( $\square ; n=4)$ and reagent grade water $(\square ; n=12)$ samples. The twelve reagent grade water samples included six seeded with 300 MPN and six seeded with 1,000 MPN of poliovirus. Error bars represent standard error.

\section{Discussion}

USEPA Method 1615 was developed for use during the Unregulated Contaminant Monitoring Regulation's third monitoring cycle (UCMR3) ${ }^{17}$ and designed primarily for measuring virus occurrence in groundwater. It shares a number of common steps with the Information Collection Rule (ICR) method, ${ }^{15,18}$ but has two minor differences. Both use quantal assays to measure virus that produce CPE on BGM cell monolayers with quantitation being based upon Most Probable Number (MPN) calculations. Method 1615 allows the use of a newer electropositive filter for concentrating virus from various water matrices and reduces the number of cell culture test vessel replicates per dilution from 20 to 10 . Both minor changes reduce overall method costs. The reduction in the number of replicates reduces labor, but results in a slightly lower detection limit. Although groundwaters are expected to have lower concentrations of virus than surface waters, ${ }^{19,20}$ the amount of sample assayed is five times that of surface water, compensating in part for the differences. The use of fewer replicates will be adequate for most surface waters, but some will require sample dilution.

Method 1615 has several critical steps and limitations. Beef extracts vary from lot to lot. Each lot should be tested for effectiveness of the virus elution and the capacity for virus concentration through the secondary concentration steps as described is supplemental materials section S2.3. The method uses precise formulas for calculating the amount of sample to inoculate onto BGM cell cultures and to determine virus titers. Inaccurate results will be generated if these formulas are not rigorously followed. Proper aseptic technique must be used for maintaining cell cultures. Uninfected BGM cell culture controls that show distress during the 14-day incubation period likely indicate problems with cell culture maintenance. Great care also must be taken during pipetting steps involved with inoculation of and medium addition to cell culture flasks to avoid cross-contamination. The quality controls described in supplemental materials Section S2 must be rigorously followed. Section S2 also provides troubleshooting advice for quality issues.

The primary mechanism of virus adsorption to electropositive filters is a charge interaction related to the strength of the positive charge on the filter and the strength of the virus' negative charge related to its isoelectric point and the $\mathrm{pH}$ of the water being tested ${ }^{21}$. Elution from filters also is 
affected by the strength of these interactions. Because they vary among virus types and even among strains within the same type, elution from the filters is not uniform. This means that any result may underestimate the actual level of virus present in environmental waters. The use of the single BGM cell line also underestimates virus occurrence. The range of enteric virus that can produce CPE in this cell line primarily is limited to polioviruses and Enterovirus $B$ species serotypes as well as some reoviruses ${ }^{14,15,22}$. Other infectious virus types will not be detected.

Poliovirus recoveries from ground and reagent grade waters met the USEPA Method 1615 performance acceptance criteria for both Performance Evaluation (PE; i.e., seeded reagent grade water samples with titers unknown to an analyst that are used to evaluate the performance of the analyst prior to the start of a study) and LFB samples (supplemental materials Table S1). The $58 \%$ recovery from groundwater using the culture procedure is similar to that reported by others using tap wate ${ }^{23,24}$. The mean recovery from the LFB samples of $111 \%$ with a coefficient of variation (CV) of $100 \%$ also met the method performance acceptance criteria even though they are higher than that observed for PE samples during the ICR. ICR mean interlaboratory recovery was $56 \%$ with a coefficient of variation $(\mathrm{CV})$ of $92 \%$ while mean intralaboratory recoveries varied from 36 to $85 \%$ (CVs 58 to 131\%; unpublished data from the ICR PE database). Higher recoveries were observed in this study for low seed LFB samples than for higher seed samples (122 versus 42\%). At the time that the ICR was being planned, it was expected that $\mathrm{PE}$ samples receiving low seed values would have lower recovery that those receiving high seeds. Similar to that observed here for the LFB samples, poliovirus recovery for ICR PE samples were 71\% (CV 100\%), 54\% (CV 69\%), and 44\% (CV $71 \%$ ) for seed values $\leq 300 \mathrm{MPN}, 300-$ $1,500 \mathrm{MPN}$, and $>1,500 \mathrm{MPN}$, respectively.

There are many methods for measuring infectious virus in water samples ${ }^{25}$. This method is significant in respect to other methods in the degree of standardization. The standardization not only includes quality and performance controls, but also uses defined volumes and formulas to ensure that all analytical laboratories perform the method identically. Without standardization, it is difficult to compare results across laboratories, and therefore standardization is essential when conducting large scale studies in multiple analytical laboratories. With the built-in standardization this method could be expanded in the future to include additional virus types and cell lines. Research is underway to provide data for inclusion of adenovirus into the method.

\section{Disclosures}

The authors have nothing to disclose.

\section{Acknowledgements}

The authors thank Dr. Mark Borchardt, U.S. Department of Agriculture, Marshfield, WI, for supplying the Sabin poliovirus serotype 3 used in this study; Mary Jean See, Nancy Schable, and Jenifer Jones of Dynamac Corporation for preparation of BGM cultures; Nichole E. Brinkman, Shannon M. Griffin, Brian R. McMinn, Eric R. Rhodes, Eunice A. Varughese, Ann C. Grimm, Sandhya U. Parshionikar, and Larry Wymer for contributions in the overall evaluation of USEPA Method 1615; Gretchen Sullivan for technical assistance; and local private well owners and utilities for allowing us to collect water samples. Although this work was reviewed by USEPA and approved for publication, it may not necessarily reflect official Agency policy. Mention of trade names or commercial products does not constitute endorsement or recommendation for use.

\section{References}

1. Bradbury, K. R. et al. Source and transport of human enteric viruses in deep municipal water supply wells. Environ. Sci. Technol. 47 (9), 4096-4103 (2013).

2. Aslan, A., Xagoraraki, I., Simmons, F. J., Rose, J. B., \& Dorevitch, S. Occurrence of adenovirus and other enteric viruses in limited-contact freshwater recreational areas and bathing waters. J. Appl. Microbiol. 111 (5), 1250-1261, (2011).

3. Begier, E. M. et al. An outbreak of concurrent echovirus 30 and coxsackievirus $A 1$ infections associated with sea swimming among a group of travelers to Mexico. Clin. Infect. Dis. 47 (5), 616-623 (2008).

4. Anderson, A. D. et al. A waterborne outbreak of Norwalk-like virus among snowmobilers-Wyoming, 2001. J. Infect. Dis. 187 (2), $303-306$ (2003).

5. Glass, R. I., Parashar, U. D., \& Estes, M. K. Norovirus gastroenteritis. N. Engl. J. Med. 361 (18), 1776-1785 (2009).

6. Khetsuriani, N., Lamonte-Fowlkes, A., Oberst, S., \& Pallansch, M. A. Enterovirus surveillance--United States, 1970-2005. MMWR Surveill. Summ. 55 (8), 1-20 (2006).

7. Sawyer, M. H. Enterovirus infections: diagnosis and treatment. Semin. Pediatr. Infect. Dis. 13 (1), $40-47$ (2002).

8. Abzug, M. J. The enteroviruses: an emerging infectious disease? The real, the speculative and the really speculative. In: Hot Topics in Infection and Immunity in Children IV. Finn, A., \& Pollard, A. J., eds, Springer (2008).

9. Harris, J. P., Edmunds, W. J., Pebody, R., Brown, D. W., \& Lopman, B. A. Deaths from norovirus among the elderly, England and Wales. Emerg. Infect. Dis. 14 (10), 1546-1552 (2008).

10. Ho, M. et al. An Epidemic of Enterovirus 71 Infection in Taiwan. New England J. Med. 341 (13), $929-935$ (1999).

11. Bae, J., \& Schwab, K. J. Evaluation of murine norovirus, feline calicivirus, poliovirus, and MS2 as surrogates for human norovirus in a model of viral persistence in surface water and groundwater. Appl. Environ. Microbiol. 74 (2), 477-484 (2008).

12. Ward, R. L., Knowlton, D. R., \& Winston, P. E. Mechanism of inactivation of enteric viruses in fresh water. Appl. Environ. Microbiol. 52 (3), 450-459 (1986).

13. Kahler, A. M., Cromeans, T. L., Roberts, J. M., \& Hill, V. R. Effects of Source Water Quality on Chlorine Inactivation of Adenovirus, Coxsackievirus, Echovirus, and Murine Norovirus. Appl. Environ. Microbiol. 76 (15), 5159-5164 (2010).

14. Dahling, D. R., \& Wright, B. A. Optimization of the BGM cell line culture and viral assay procedures for monitoring viruses in the environment. Appl. Environ. Microbiol. 51 (4), 790-812 (1986).

15. Fout, G. S., Schaefer, F. W., 3rd, Messer, J. W., Dahling, D. R., \& Stetler, R. E. ICR Microbial Laboratory Manual. EPA/600/R-95/178, U.S. Environmental Protection Agency, I.1-ApD-23 (1996). 
16. Cashdollar, J. L. et al. Development and Evaluation of EPA Method 1615 for Detection of Enterovirus and Norovirus in Water. Appl. Environ. Microbiol. 79 (1), 215-223, doi: 10.1128/aem.02270-12 (2013).

17. USEPA. 40 CFR Parts 141 and 142 Revisions to the Unregulated Contaminant Monitoring Regulation (UCMR3) for Public Water Systems; Final Rule. Federal Register. 77 (85), 26072-26101 (2012).

18. USEPA. 40 CFR Part 141 National Primary Drinking Water Regulations: Monitoring Requirements for Public Drinking Water Supplies; Final Rule. Federal Register. 61 (94), 24353-24388 (1996).

19. Shaw, S., Regli, S., \& Chen, J. Virus occurrence and health risks in drinking water. In: Information Collection Rule Data Analysis. McGuire, M. J., McLain, J. L., \& Obolensky, A., eds, AWWA Research Foundation and American Water Works Association, 437-462 (2002).

20. Lieberman, R. J. et al. Microbial monitoring of vulnerable public groundwater supplies. American Water Works Association,Denver, CO, (2002).

21. Lukasik, J., Scott, T. M., Andryshak, D., \& Farrah, S. R. Influence of salts on virus adsorption to microporous filters. Appl. Environ. Microbiol. 66 (7), 2914-2920 (2000).

22. Sedmak, G., Bina, D., \& MacDonald, J. Assessment of an enterovirus sewage surveillance system by comparison of clinical isolates with sewage isolates from milwaukee, wisconsin, collected august 1994 to december 2002. Appl. Environ. Microbiol. 69 (12), $7181-7187$ (2003).

23. Ikner, L. A., Soto-Beltran, M., \& Bright, K. R. New method using a positively charged microporous filter and ultrafiltration for concentration of viruses from tap water. Appl. Environ. Microbiol. 77 (10), 3500-3506 (2011).

24. Karim, M. R., Rhodes, E. R., Brinkman, N., Wymer, L., \& Fout, G. S. New electropositive filter for concentrating enteroviruses and noroviruses from large volumes of water. Appl. Environ. Microbiol. 75 (8), 2393-2399 (2009).

25. Cashdollar, J. L., \& Wymer, L. Methods for primary concentration of viruses from water samples: a review and meta-analysis of recent studies. J Appl Microbiol. 115 (1), 1-11 (2013). 